\title{
Transgenic overexpression of insulin-like growth factor I prevents streptozotocin-induced cardiac contractile dysfunction and $\beta$-adrenergic response in ventricular myocytes
}

\author{
F L Norby, N S Aberle II, J Kajstura ${ }^{1}$, P Anversa ${ }^{1}$ and J Ren \\ Division of Pharmaceutical Sciences, University of Wyoming College of Health Sciences, Laramie, Wyoming 82071, USA \\ ${ }^{1}$ Department of Medicine, Cardiovascular Research Institute, New York Medical College, Valhalla, New York 10595, USA \\ (Requests for offprints should be addressed to J Ren; Email: jren@uwyo.edu)
}

\begin{abstract}
Diabetic cardiomyopathy is characterized by cardiac dysfunction and altered level/function of insulin-like growth factor I (IGF-I). Both endogenous and exogenous IGF-I have been shown to effectively alleviate diabetes-induced cardiac dysfunction and oxidative stress. This study was designed to examine the effect of cardiac overexpression of IGF-I on streptozotocin (STZ)-induced cardiac contractile dysfunction in mouse myocytes. Both IGF-I heterozygous transgenic mice and their wild-type FVB littermates were made diabetic with a single injection of STZ $(200 \mathrm{mg} / \mathrm{kg}$, i.p.) and maintained for 2 weeks. The following mechanical indices were evaluated in ventricular myocytes: peak shortening (PS), time-to-PS (TPS), time-to-90\% relengthening $\left(\mathrm{TR}_{90}\right)$ and maximal velocity of shortening/ relengthening $( \pm \mathrm{dL} / \mathrm{dt})$. Intracellular $\mathrm{Ca}^{2+}$ was evaluated as resting and peak intracellular $\mathrm{Ca}^{2+}$ levels, $\mathrm{Ca}^{2+}$-induced
\end{abstract}

$\mathrm{Ca}^{2+}$ release and intracellular $\mathrm{Ca}^{2+}$ decay rate (tau). STZ led to hyperglycemia in FVB and IGF-I mice. STZ treatment prolonged TPS and $\mathrm{TR}_{90}$, reduced $\mathrm{Ca}^{2+}$ induced $\mathrm{Ca}^{2+}$ release, increased resting intracellular $\mathrm{Ca}^{2+}$ levels and slowed tau associated with normal PS and $\pm \mathrm{dL} / \mathrm{dt}$. All of which, except the elevated resting intracellular $\mathrm{Ca}^{2+}$, were prevented by the IGF-I transgene. In addition, myocytes from STZ-treated FVB mice displayed an attenuated contractile response to the $\beta$-adrenergic agonist isoproterenol, which was restored by the IGF-I transgene. Contractile response to the $\alpha$-adrenergic agonist phenylephrine and angiotensin II was not affected by either STZ treatment or IGF-I. These results validate the beneficial role of IGF-I in diabetic cardiomyopathy, possibly due to an improved $\beta$-adrenergic response.

Journal of Endocrinology (2004) 180, 175-182

\section{Introduction}

Diabetes mellitus is associated with an elevated cardiovascular morbidity and mortality largely due to diabetesrelated structural and functional damage in the heart (Fein 1990, Ren et al. 1999). Diabetic cardiomyopathy is believed to contribute predominantly to the enhanced likelihood of cardiac death in diabetics independent of macro/microvascular complications (Fein 1990). The most prominent defects of diabetic cardiomyopathy include compromised ventricular functions such as prolonged duration of contraction and relaxation and reduced cardiac compliance (Fein 1990, Ren \& Davidoff 1997, Ren \& Bode 2000). The etiology of diabetic cardiomyopathy is rather complex and may involve metabolic derangements, depressed autonomic function and abnormalities in certain hormones or proteins that regulate intracellular ion homeostasis, particularly $\mathrm{Ca}^{2+}$ (Fein 1990, Rodrigues et al. 1998, Norby et al. 2002). Nevertheless, either endogenous overexpression or exogenous administration of a key cardiac surviving factor, insulin-like growth factor I (IGF-I) has exhibited beneficial effects on diabetes-induced cardiac mechanical dysfunction and oxidative damage (Kajstura et al. 2001, Norby et al. 2002). IGF-I, an insulin analogue and replacement for glucose control in diabetes, promotes cardiac growth and myocardial contraction, improves hemodynamics and energy metabolism, and protects the heart against apoptosis induced by ischemia or oxidative stress (Wang et al. 1998, Leri et al. 1999, Ren et al. 1999, Kajstura et al. 2001). The cardiac protective capacity of IGF-I is believed to be mediated through the phosphatidylinositol-3 (PI-3)kinase-mediated activation of the serine-threonine kinase Akt (Coffer et al. 1998, Ren et al. 2003). Resistance to IGF-I-induced cardiac response has been shown in chemically induced and genetically predisposed diabetic conditions, or in vitro hyperglycemic conditions (Ren et al. 1999, Ren 2000, Ren et al. 2003). In addition, altered cardiac IGF-I/IGF-I receptor levels have been reported in diabetic patients and experimental animals, suggesting a critical role of IGF-I in diabetic cardiomyopathy (Bornfeldt et al. 1992, Bereket et al. 1999, Duan et al. 2003). 
To further test the role of transgenic overexpression of IGF-I on cardiomyocyte function in diabetes, experimental diabetes was induced with a single injection of streptozotocin (STZ) in mice heterozygous for the IGF-I transgene or their wild-type FVB littermates (Reiss et al. 1996). State-of-the-art video-based edge-detection and intracellular $\mathrm{Ca}^{2+}$ fluorescent imaging techniques were used to examine the mechanical properties in isolated ventricular myocytes. Since our goal was to characterize the impact of IGF-I on the development of diabetic cardiomyopathy, using cardiac specific IGF-I transgenic mice should help to avoid any undesired systemic effects as a result of exogenous IGF-I administration, which may have secondary effects on the hearts.

\section{Materials and Methods}

\section{Experimental animals}

The experiments described here were approved by the Institutional Animal Care and Use Committees of University of North Dakota (Grand Forks, ND, USA) and University of Wyoming (Laramie, WY, USA). All animal procedures were in accordance with $\mathrm{NIH}$ animal care standards.

Eight to ten week-old male IGF-I heterozygous transgenics and their weight-matched wild-type FVB littermates (offspring from male heterozygous IGF-I transgenic mice cross to female FVB mice) were injected with streptozotocin (STZ, $200 \mathrm{mg} / \mathrm{kg}$, i.p.). The pigmentation of fur color (light brown) was used as a marker for heterozygous IGF-I or wild-type FVB mouse identification as described in Reiss et al. (1996). Mice were then maintained for 2 weeks with free access to standard lab chow and tap water. Blood glucose levels were measured weekly using a glucose monitor (Accu-ChekII, model 792, Boehringer Mannheim Diagnostics, Indianapolis, IN, USA).

\section{Ventricular myocytes isolation procedures}

Hearts were rapidly removed from anesthetized mice and immediately mounted on a temperature-controlled $\left(37^{\circ} \mathrm{C}\right)$ Langendorff perfusion system. After perfusion with modified $\left(\mathrm{Ca}^{2+}\right.$ free) Tyrode solution $(\mathrm{pH} 7 \cdot 4$, containing (in $\mathrm{mM}$ ): $135 \mathrm{NaCl}, 4 \mathrm{KCl}, 1 \mathrm{MgCl}_{2}, 10$ HEPES, $0.33 \mathrm{NaH}_{2} \mathrm{PO}_{4}, 10$ glucose, 10 butanedione monoxime, and gassed with $5 \% \mathrm{CO}_{2}-95 \% \mathrm{O}_{2}$ ) for $2 \mathrm{~min}$, the heart was digested for $10 \mathrm{~min}$ with $0.9 \mathrm{mg} / \mathrm{ml}$ collagenase D (Boehringer Mannheim Biochemicals) in modified Tyrode solution. The digested heart was then removed from the cannula and the left ventricle was cut into small pieces in the modified Tyrode solution. These pieces were gently agitated and the pellet of cells was resuspended in modified Tyrode solution and allowed to settle for another $20 \mathrm{~min}$ at room temperature during which time extracellular $\mathrm{Ca}^{2+}$ was added incrementally back to $1.20 \mathrm{mM}$. Isolated myocytes were used for experiments within $8 \mathrm{~h}$ of isolation. Only rod-shaped myocytes with clear edges were selected for recording of mechanical properties and intracellular $\mathrm{Ca}^{2+}$ transients as described in Duan et al. (2003).

\section{Cell shortening/relengthening}

Mechanical properties of ventricular myocytes were assessed using a SoftEdge MyoCam system (IonOptix Corporation, Milton, MA, USA) (Duan et al. 2003). In brief, cells were placed in a Warner chamber mounted on the stage of an inverted microscope (Olympus IX-70, Olympus Optical Corporation, Tokyo, Japan) and superfused $\left(\sim 1 \mathrm{ml} / \mathrm{min}\right.$ at $\left.25^{\circ} \mathrm{C}\right)$ with a buffer containing (in $\mathrm{mM}): 131 \mathrm{NaCl}, 4 \mathrm{KCl}, 1 \mathrm{CaCl}_{2}, 1 \mathrm{MgCl}_{2}, 10$ glucose, $10 \mathrm{HEPES}$ at $\mathrm{pH} 7 \cdot 4$. The cells were field stimulated with suprathreshold voltage at a frequency of $0.5 \mathrm{~Hz}, 3 \mathrm{msec}$ duration, using a pair of platinum wires placed on opposite sides of the chamber connected to a FHC stimulator (FHC Incorporation, Bowdoinham, ME, USA). The myocyte being studied was displayed on the computer monitor using an IonOptix MyoCam camera. SoftEdge software was used to capture changes in cell length during shortening and relengthening.

\section{Intracellular fluorescence measurement}

A separate cohort of murine myocytes was loaded with fura-2/AM $(0.5 \mu \mathrm{M})$ for $10 \mathrm{~min}$ and fluorescence measurements were recorded with a dual-excitation fluorescence photomultiplier system (Ionoptix) as described in Duan et al. (2003). Myocytes were placed on an Olympus IX-70 inverted microscope and imaged through a Fluor $\times 40$ oil objective. Cells were exposed to light emitted by a $75 \mathrm{~W}$ lamp and passed through either a 360 or a $380 \mathrm{~nm}$ filter (bandwidths were $\pm 15 \mathrm{~nm}$ ), while being stimulated to contract at $0.5 \mathrm{~Hz}$. Fluorescence emissions were detected between 480 and $520 \mathrm{~nm}$ by a photomultiplier tube after first illuminating the cells at $360 \mathrm{~nm}$ for $0.5 \mathrm{sec}$ then at $380 \mathrm{~nm}$ for the duration of the recording protocol $(333 \mathrm{~Hz}$ sampling rate). The $360 \mathrm{~nm}$ excitation scan was repeated at the end of the protocol and qualitative changes in intracellular $\mathrm{Ca}^{2+}$ concentration $\left(\left[\mathrm{Ca}^{2+}\right]_{\mathrm{i}}\right)$ were inferred from the ratio of the fluorescence intensity at two wavelengths.

\section{Statistical analyses}

Nine mice were used in each of the four groups. Windows of 10-18, 6-10, and 3-5 cardiac myocytes were evaluated for cell shortening, intracellular $\mathrm{Ca}^{2+}$ transient and drug response, respectively, from each mouse. Mechanical results from multiple myocytes were pooled and shown as 
Table 1 General features of normal or STZ-induced diabetic FVB and IGF-1 mice

\begin{tabular}{lccc} 
Mouse group & & \\
\hline FVB $(n=9)$ & IGF-1 $(n=9)$ & FVB-STZ $(n=9)$ & IGF-STZ $(n=9)$ \\
\cline { 2 - 2 } $28 \cdot 3 \pm 1 \cdot 6$ & $28 \cdot 7 \pm 1 \cdot 2$ & $23 \cdot 5 \pm 1 \cdot 2^{* *}$ & $26 \cdot 2 \pm 1 \cdot 2$ \\
$178 \pm 15$ & $284 \pm 33^{*}$ & $191 \pm 17$ & $199 \pm 20$ \\
$6 \cdot 20 \pm 0 \cdot 26$ & $10 \cdot 71 \pm 0 \cdot 39^{\#}$ & $8 \cdot 36 \pm 0 \cdot 91^{\#}$ & $7 \cdot 73 \pm 0 \cdot 84^{\#}$ \\
$1 \cdot 418 \pm 0 \cdot 078$ & $1 \cdot 564 \pm 0 \cdot 103$ & $1 \cdot 34 \pm 0 \cdot 111$ & $1 \cdot 404 \pm 0 \cdot 112$ \\
$51 \cdot 23 \pm 2 \cdot 14$ & $54 \cdot 19 \pm 1 \cdot 45$ & $56 \cdot 69 \pm 2 \cdot 31$ & $53 \cdot 67 \pm 3 \cdot 14$ \\
$0 \cdot 478 \pm 0 \cdot 035$ & $0 \cdot 549 \pm 0 \cdot 042$ & $0 \cdot 476 \pm 0 \cdot 021$ & $0 \cdot 618 \pm 0 \cdot 060^{\#}$ \\
$17 \cdot 12 \pm 0 \cdot 77$ & $18 \cdot 97 \pm 0 \cdot 84$ & $20 \cdot 52 \pm 1 \cdot 04^{\#}$ & $23 \cdot 77 \pm 1 \cdot 90^{* *}$ \\
$6 \cdot 08 \pm 0 \cdot 55$ & $5 \cdot 89 \pm 0 \cdot 34$ & $15 \cdot 79 \pm 1 \cdot 24^{* *}$ & $23 \cdot 95 \pm 2 \cdot 13^{* *}$
\end{tabular}

HW, heart weight; LW, liver weight; KW, kidney weight. Means \pm S.E.M., ${ }^{*} P<0 \cdot 05$ vs all other groups, ${ }^{* *} P<0 \cdot 05$ vs FVB and IGF-1 groups (without STZ treatment), ${ }^{\#} P<0.05$ vs FVB group (without STZ treatment), $n$, number of animals.

a single data point in each mouse. For each experimental series, data are presented as means \pm S.E.M. Statistical significance $(P<0 \cdot 05)$ for each variable was estimated by ANOVA or t-test, where appropriate (SYSTAT, Inc., Evanston, IL, USA). A Dunnett's test was used for post hoc analysis.

\section{Results}

\section{General features of the experimental animals}

Short-term (2 weeks) diabetes significantly reduced the body weight gain in FVB but not IGF-I mice (although there was a trend of body weight loss). STZ treatment significantly increased blood glucose levels in both FVB and IGF-I mice. Transgenic overexpression of IGF-I itself significantly enhanced heart weight and heart-to-body weight ratio. STZ treatment failed to affect absolute heart weight but significantly enhanced heart-to-body weight and kidney-to-body weight ratio in both mice groups. The IGF-I transgene did not elicit any further effect on organ-to-body weight ratio in addition to those induced by STZ treatment. The liver weights were not affected by either diabetes or the IGF-I transgene (Table 1).

\section{Cell shortening and relengthening properties of myocytes from FVB and IGF-I mice}

As shown in Fig. 1, transgenic overexpression of IGF-I did not significantly affect any of the mechanical parameters tested. There was no significant difference in the resting cell length (CL) of ventricular myocytes among normal or diabetic FVB or IGF-I mice. Peak shortening (PS) amplitude normalized to CL was similar in ventricular myocytes among all 4 groups examined. Myocytes from the diabetic FVB mice demonstrated significantly prolonged time-topeak shortening (TPS) and time-to-90\%-relengthening $\left(\mathrm{TR}_{90}\right)$ compared with those from all other groups, which is consistent with our previous findings (Duan et al. 2003,
Ye et al. 2003). Transgenic overexpression of IGF-I in the heart blunted the diabetes-induced prolongation of TPS and $\mathrm{TR}_{90}$. Neither the maximal velocity of shortening $(+\mathrm{dL} / \mathrm{dt})$ nor relengthening $(-\mathrm{dL} / \mathrm{dt})$ was significantly affected by STZ treatment or IGF-I transgene.

\section{Intracellular $\mathrm{Ca}^{2+}$ transient properties in FVB and IGF-I mouse myocytes}

We used the membrane permeant form of fura-2/AM to evaluate the properties of intracellular $\mathrm{Ca}^{2+}$ transients in cardiomyocytes from normal or diabetic FVB and IGF-I mice. The time course of the fluorescence signal decay was fitted by a single exponential equation, and the time constant (tau) was used as a measure of the cytoplasmic $\mathrm{Ca}^{2+}$ clearing rate. The fluorescence measurements revealed that the IGF-I transgene itself significantly reduced the baseline fura-2 fluorescent intensity (representing resting intracellular $\mathrm{Ca}^{2+}$ level) without affecting the peak intracellular $\mathrm{Ca}^{2+}$ level in response to electrical stimulus, the $\mathrm{Ca}^{2+}$-induced $\mathrm{Ca}^{2+}$ release (the difference between peak and resting intracellular $\mathrm{Ca}^{2+}$ levels) and intracellular $\mathrm{Ca}^{2+}$ decay rate. Interestingly, diabetes elevated resting intracellular $\mathrm{Ca}^{2+}$, reduced $\mathrm{Ca}^{2+}$-induced $\mathrm{Ca}^{2+}$ release and slowed the intracellular $\mathrm{Ca}^{2+}$ clearing rate without affecting the peak intracellular $\mathrm{Ca}^{2+}$ level. All of these diabetes-induced defects in intracellular $\mathrm{Ca}^{2+}$ homeostasis, with the exception of resting intracellular $\mathrm{Ca}^{2+}$, were effectively prevented by the cardiac overexpression of IGF-I transgene (Fig. 2).

\section{Effect of phenylephrine, isoproterenol and angiotensin II on myocyte PS}

Altered function of adrenergic and angiotensin systems has been documented in diabetes-associated heart dysfunction (Roth et al. 1995, Kajstura et al. 2001, Ye et al. 2003). To compare the function of adrenergic and angiotensin systems in myocytes from normal or diabetic FVB and IGF-I 
(a)

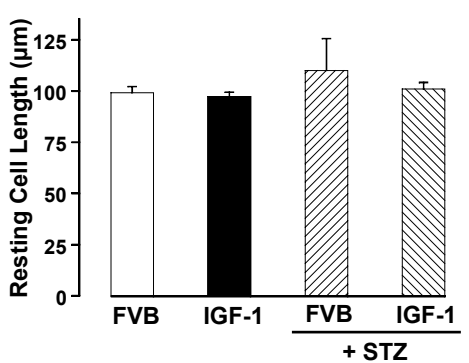

(c)

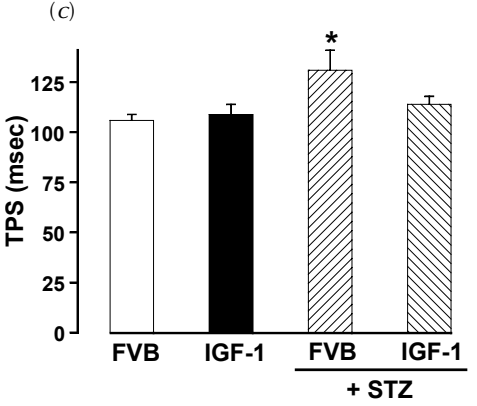

(e)

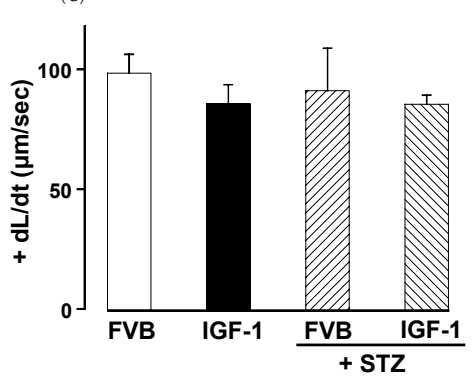

(b)
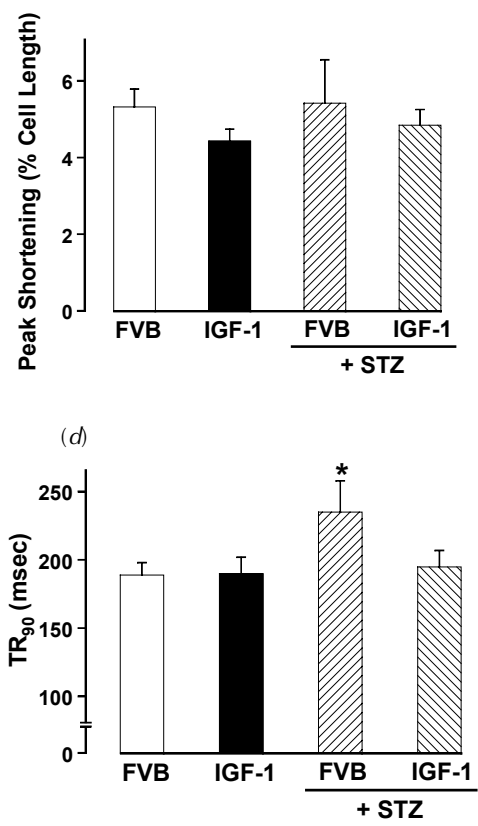

(f)

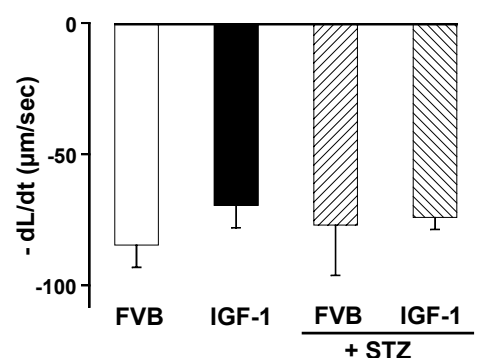

Figure 1 Contractile properties of ventricular myocytes from normal or STZ-induced diabetic FVB and IGF-I transgenic mice. (a) Resting cell length. (b) Peak shortening (PS as $\%$ of resting cell length). (c) Time-to-peak shortening (TPS). (d) Time-to-90\% relengthening $\left(T_{90}\right)$. (e) Maximal velocity of shortening $(+d L / d t)$. $(f)$ Maximal velocity of relengthening $(-\mathrm{dL} / \mathrm{dt})$. Means \pm S.E.M., $n=9$ mice per group $\left(10-18\right.$ myocytes in each mouse), ${ }^{\star} P<0.05$ vs all other groups.

mouse hearts, ventricular myocytes were exposed to the $\alpha$-adrenergic agonist phenylephrine, the $\beta$-adrenergic agonist isoproterenol and angiotensin II for $10 \mathrm{~min}$ each. Figure 3 showed that phenylephrine $(10$ and $100 \mu \mathrm{M})$ significantly depressed PS, which was comparable among all groups. The isoproterenol $(1$ and $10 \mu \mathrm{M})$-induced positive response in PS, seen in three of the groups, was blunted in myocytes from the FVB-STZ diabetic mice. However, this depressed response was restored by the IGF-I transgene. Finally, angiotensin II (10 and $100 \mathrm{nM})$ exerted similar patterns of depression in PS in myocytes of normal or diabetic FVB and IGF-I groups. The IGF-I transgene itself did not significantly alter the myocyte contractile response to phenylephrine, isoproterenol or angiotensin II.

\section{Discussion}

The present study, using cardiac-specific IGF-I overexpression transgenic mice, confirmed our earlier observations regarding the beneficial effects of IGF-I in diabetic cardiac dysfunction (Kajstura et al. 2001, Norby et al. 2002). In addition, we found that the IGF-Iassociated beneficial effects on diabetic heart dysfunction may be related, at least in part, to the improved $\beta$-adrenergic responsiveness in diabetes. Our results did not favor the notion that the $\alpha$-adrenergic or angiotensin II-associated cardiac contractile response plays a major role in diabetes-induced cardiomyocyte dysfunction or the IGF-I-associated cardiac protection against diabetes. 
(a)

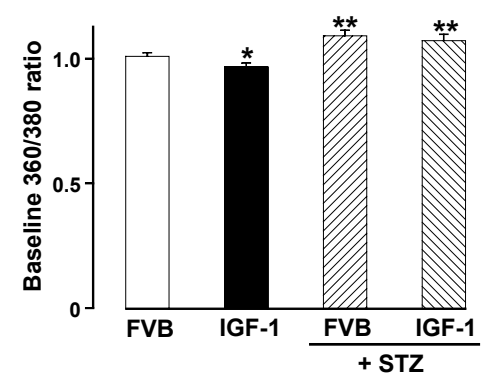

(c)

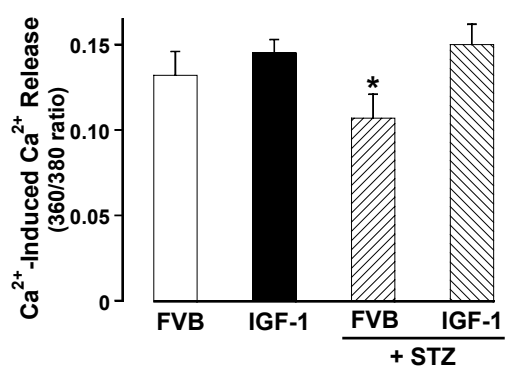

(b)

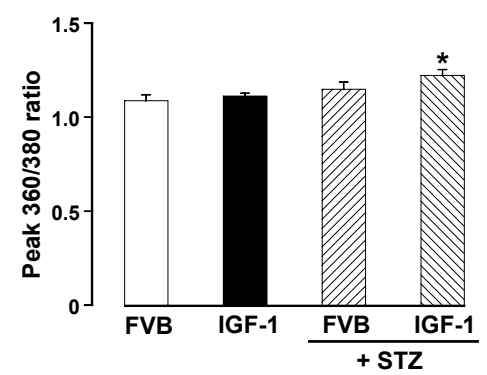

(d)

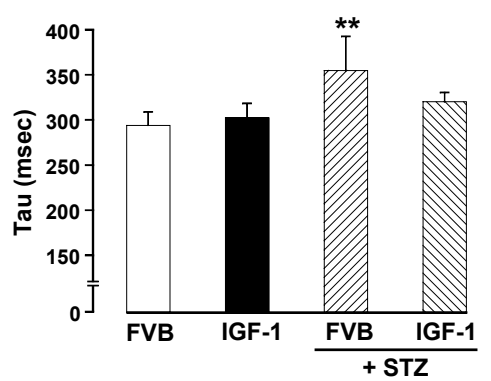

Figure 2 Intracellular $\mathrm{Ca}^{2+}$ properties of ventricular myocytes from normal or STZ-induced FVB and IGF-I transgenic mice. (a) Resting intracellular $\mathrm{Ca}^{2+}$ levels. (b) Peak intracellular $\mathrm{Ca}^{2+}$ levels. (c) Increase in intracellular $\mathrm{Ca}^{2+}$ in response to electrical stimuli.

(d) Intracellular $\mathrm{Ca}^{2+}$ transient decay rate (tau). Means \pm S.E.M., $n=9$ mice per group (6-10 myocytes in each mouse), ${ }^{*} P<0.05$ vs all other groups, ${ }^{*} P<0.05$ vs FVB and IGF-I groups (without STZ treatment).

Prolonged contraction and relaxation duration are the hallmarks of diabetic cardiomyopathy (Ren \& Davidoff 1997, Ren \& Bode 2000, Duan et al. 2003). Somewhat similar to our earlier observations, the current study demonstrated prolonged duration of TPS and $\mathrm{TR}_{90}$ associated with normal PS amplitude and maximal rate of shortening and relengthening $( \pm \mathrm{dL} / \mathrm{dt})$ in STZ-induced diabetes (Ren \& Davidoff 1997, Ren \& Bode 2000, Duan et al. 2003). These mechanical dysfunctions are consistent with our current findings of impaired intracellular $\mathrm{Ca}^{2+}$ homeostasis shown as reduced intracellular $\mathrm{Ca}^{2+}$ clearing rate and the $\mathrm{Ca}^{2+}$-induced intracellular $\mathrm{Ca}^{2+}$ release in STZ-induced diabetic mouse ventricular myocytes. It is worth pointing out that the mechanical as well as intracellular $\mathrm{Ca}^{2+}$ defects observed in STZ diabetic mice are similar to those found in diabetic rat models (Ren \& Davidoff 1997, Ren \& Bode 2000). Several mechanisms have been postulated for these mechanical and intracellular $\mathrm{Ca}^{2+}$ defects. It has been reported that the depressed rate of shortening may be associated with diabetes-induced shifts in contractile protein isoforms, such as the shift of myosin heavy chain isoform from the fast type $\left(\mathrm{V}_{1}\right)$ to the slow type $\left(\mathrm{V}_{3}\right)$ (Dillmann 1989). Diabetes has also been demonstrated to significantly reduce the myofilament $\mathrm{Ca}^{2+}$ sensitivity (Hofmann et al. 1995, Ren \& Bode 2000).
Finally, the slowed intracellular $\mathrm{Ca}^{2+}$ decay and prolonged duration of relaxation may simply be a consequence of impaired sarco(endo)plasmic reticulum $\mathrm{Ca}^{2+}-\mathrm{ATPase}$ (SERCA) and $\mathrm{Na}^{+} / \mathrm{Ca}^{2+}$ exchanger function (Dillmann 1989, Chattou et al. 1999). Although the mechanism behind the higher peak $\mathrm{Ca}^{2+}$ response in the IGF-STZ mouse group (Fig. $2 \mathrm{~b}$ ) is not known, a synergistic effect between the IGF-I transgene and short-term STZ treatment may be speculated. Short-term STZ treatment (2 weeks) may transiently upregulate certain intracellular $\mathrm{Ca}^{2+}$ regulatory proteins through a compensatory mechanism to reconcile the detrimental cardiac effect of diabetes (Depre et al. 2000). While the compensatory mechanism itself may not be sufficient for any effect on intracellular $\mathrm{Ca}^{2+}$ handling, it may certainly be synergized with IGF-I, which is known to promote cardiac intracellular $\mathrm{Ca}^{2+}$ homeostasis (Ren et al. 1999), to significantly enhance the peak $\mathrm{Ca}^{2+}$ response in myocytes from IGF-STZ mice.

Our present study revealed that cardiac transgenic overexpression of IGF-I may prevent the diabetes-induced cardiac mechanical dysfunction at the cellular level and protect the $\beta$-adrenergic response blunted by STZ diabetic treatment. IGF-I is known to facilitate glucose metabolism, lower insulin levels, improve insulin 
(a) $\square$ FVB $\square$ IGF-1 $\square$ FVB-STZ $\square$ IGF-STZ

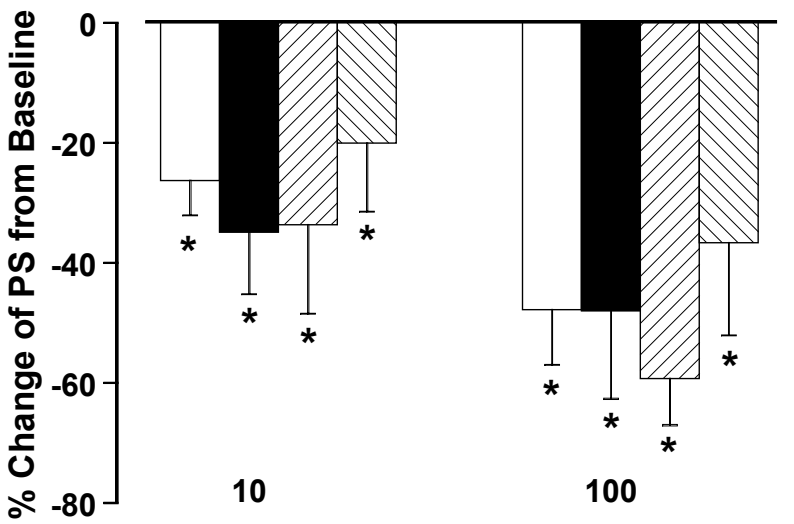

Phenylephrine $(\mu \mathrm{M})$
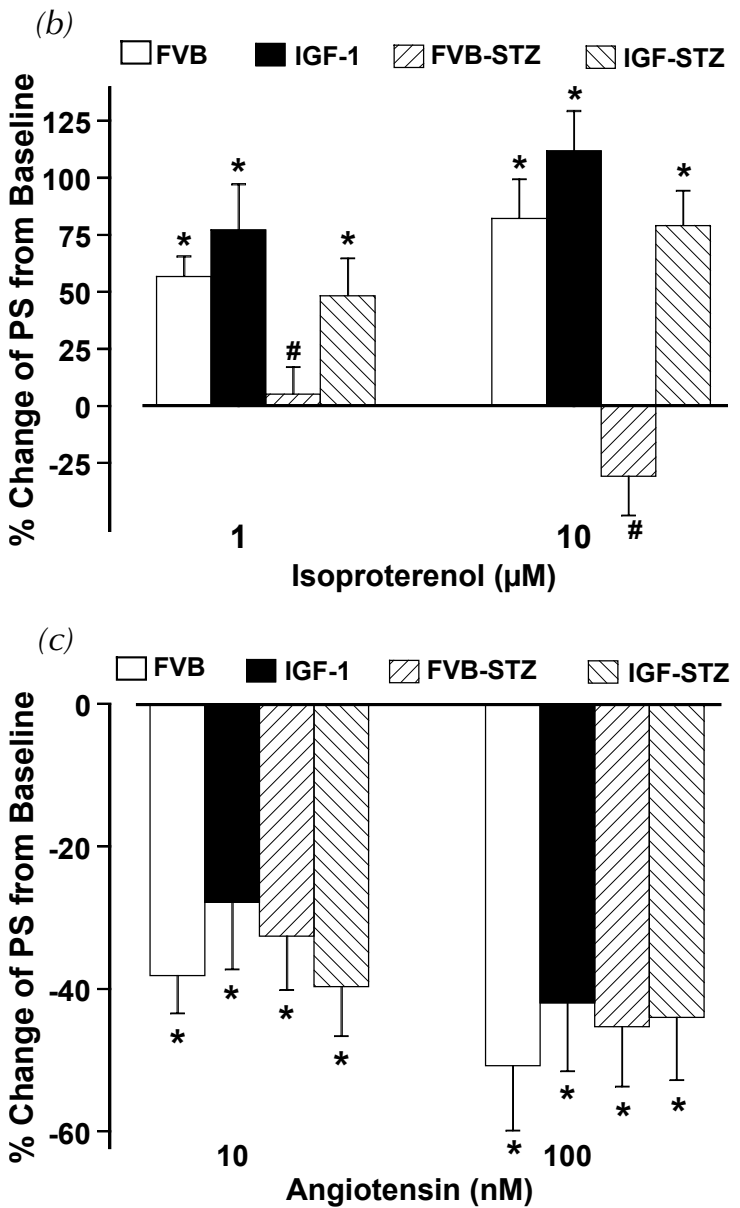

Figure 3 Concentration-dependent effects of (a) phenylephrine (10 and $100 \mu \mathrm{M}),(b)$ isoproterenol $(1$ and $10 \mu \mathrm{M})$ and (c) angiotensin II (10-100 $\mathrm{nM})$ on peak cell shortening (PS) in myocytes from normal or STZ-induced FVB and IGF-I transgenic mice. Actual data points were obtained by normalizing PS amplitude to PS value of the respective control. Means \pm S.E.M., $n=9$ mice per group ( $3-5$ myocytes in each mouse), ${ }^{*} P<0.05$ vs baseline, ${ }^{\#} P<0.05$ vs all other groups. sensitivity and lipid profile (Ren et al. 1999, Samarel 2002), suggesting both a physiological role and therapeutic potential. The rapid onset, the long duration of effect, and the relatively modest magnitude of its action compared with other endogenous substances suggest that if IGF-I has an acute cardioregulatory role, it may contribute to the modulation of the inotropic responsiveness of the myocardium over a time frame of minutes to hours (Ren et al. 1999, Samarel 2002). In addition to its beneficial effect on glucose transport and lipid metabolism, other scenarios have been speculated for IGF-I-induced cardiac protection against stress such as diabetes. IGF-I has been demonstrated to directly regulate membrane ionic channels responsible for cardiac excitation-contraction coupling (Guo et al. 1997, Solem \& Thomas 1998). Recent evidence suggested that IGF-I-induced cardiac protection against diabetes may involve attenuated p53 function, angiotensin II production and angiotensin receptor activation (Kajstura et al. 2001). Our recent study also suggested that IGF-I may rescue the diabetes-induced reduction in SERCA protein abundance (Norby et al. 2002). Data from this study suggested that the STZ treatment failed to elicit any further cardiac hypertrophic response in IGF-I transgenic mice. The higher cardiac mass and heart-to-body weight ratio in the IGF-I transgenic mice is consistent with our earlier reports and is likely to be due to a hyperplastic response of myocytes to this growth factor (Reiss et al. 1996, Welch et al. 2002). The fact that STZ treatment failed to produce any further cardiac hypertrophic response indicates that ventricular remodeling in response to IGF-I may be capable of offsetting the detrimental effect of STZ on cardiac structure and thus offer protection. Although it can be argued that the STZ treatment and IGF-I transgene may 'mask' each other in the cardiac hypertrophic response, the fact that STZ and IGF-I induce cardiac hypertrophy through completely different mechanisms makes this 'masking' hypothesis rather unlikely. STZ is believed to cause cardiac hypertrophy largely through a genomic adaptive or maladaptive process (Depre et al. 2000).

IGF-I has been demonstrated to facilitate cardiac DNA and protein synthesis, reduce protein degradation, and participate in early neonatal cardiomyocyte proliferation and maturation (see Ren et al. 1999 for review). IGF-I possesses multiple beneficial effects on cardiac structure and function such as normalization of heart mass, hemodynamics and apoptosis. IGF-I expression acts as a proliferative stimulus to enhance myocyte number through cellular replication (Reiss et al. 1996, Welch et al. 2002). In transgenic mice overexpressing IGF-I in myocardium, total heart weight is increased by $50 \%$ (consistent with our current study) and the myocyte number is increased by $20-50 \%$ (Reiss et al. 1996). On the contrary, IGF-I deficiency may lead to cardiac atrophy and impaired cardiac function (Isgaard et al. 1999, Ren \& Brown-Borg 2002). These beneficial effects of IGF-I suggest the crucial 
role of IGF-I in cardiac morphology and ventricular function.

Perhaps the most interesting result from our current study is the protected $\beta$-adrenergic responsiveness in diabetic myocytes with concurrent IGF-I overexpression, suggesting that protection of $\beta$-adrenergic responsiveness may play a role in IGF-I-induced cardiac protection. Our data is consistent with previous findings that isoproterenol increases the contraction amplitude and the velocities of contraction and relaxation in ventricular myocytes (Lefroy et al. 1996). Inconsistent observations have been documented regarding the impact of diabetes on the $\beta$-adrenergic system. Although $\beta$-adrenergic receptor number may not be depressed by diabetes, alteration in certain post-receptor mechanisms such as G-protein and adenylate cyclase are speculated to be responsible for attenuated cardiac $\beta$-adrenergic responsiveness in diabetes (Roth et al. 1995). The mechanism by which IGF-I restores the depressed $\beta$-adrenergic response in diabetes is largely unknown at this time. However, it has been shown that IGF-I directly stimulates phosphorylation of the $\beta$-adrenergic receptor in vivo on sites distinct from those phosphorylated in response to insulin (Karoor \& Malbon 1996).

Our study did not favor any role for angiotensin II and $\alpha$-adrenergic systems in either diabetes-induced cardiomyocyte dysfunction or the IGF-I-mediated improvement of cardiac mechanical function. Angiotensin II and phenylephrine are receptor agonists with similar signaling mechanisms via acceleration of cardiac phosphoinositide hydrolysis. The regulation of myocardial contractility by these receptor agonists has exhibited a wide range of species-dependent variation. While angiotensin II fails to affect contraction in rat, guinea pigs, or human atrial/ ventricular myocytes (Lefroy et al. 1996), it produces a sustained negative response in adult mice (Sekine et al. 1999). Both angiotensin II and phenylephrine have been shown to inhibit cell shortening but not intracellular $\mathrm{Ca}^{2+}$ transients through a protein kinase C-dependent pathway in mouse cardiomyocytes (Sakurai et al. 2002). Treatment of mouse papillary muscles with phenylephrine resulted in a sustained reduction in cardiac contractility, whereas a positive inotropic effect occurred in rat papillary muscles (Nishimaru et al. 2001, Montgomery et al. 2002).

\section{Experimental limitations}

Although our study provided evidence that the $\beta$-adrenergic response is likely to be protected by the IGF-I transgene under diabetes, we were unable to measure the circulating catecholamine levels and the cardiac $\beta$-adrenergic receptor binding properties in diabetic mice carrying IGF-I transgene. Further study is warranted to provide more precise information regarding the role of IGF-I on the adrenergic system in diabetes.
In summary, our findings revealed impaired cardiac contractile function representative of diabetic cardiomyopathy in STZ-induced diabetic mouse hearts, an effect which can be ablated with cardiac overexpression of IGF-I. Our data suggest the improved $\beta$-adrenergic response may play a role in the improved mechanical function elicited by IGF-I. Given what we know about the ability of IGF-I to promote cell survival and cardiac performance (Ren et al. 1999, Samarel 2002), the in-depth mechanism of action and clinical value of employing IGF-I in the prevention and treatment of diabetic cardiomyopathy warrants further investigation.

\section{Funding}

This work was supported in part by grants from American Diabetes Association (7-00-RA-21), NIH/NIA 1 R03 AG21324-01, North Dakota Max Baer Heart Fund and North Dakota Experimental Program to Stimulated Competitive Research (EPSCoR) to JR, NIH/HL-66923 (to P A) and NIH/HL-65573 (to J K).

\section{References}

Bereket A, Lang CH \& Wilson TA 1999 Alterations in the growth hormone-insulin-like growth factor axis in insulin dependent diabetes mellitus. Hormone and Metabolic Research 31 172-181.

Bornfeldt KE, Skottner A \& Arnqvist HJ 1992 In-vivo regulation of messenger RNA encoding insulin-like growth factor-I (IGF-I) and its receptor by diabetes, insulin and IGF-I in rat muscle. Journal of Endocrinology 135 203-211.

Chattou S, Diacono J \& Feuvray D 1999 Decrease in sodium-calcium exchange and calcium currents in diabetic rat ventricular myocytes. Acta Physiologica Scandinavica 166 137-144.

Coffer PJ, Jin J \& Woodgett JR 1998 Protein kinase B (c-Akt): a multifunctional mediator of phosphatidylinositol 3-kinase activation. Biochemical Journal 335 1-13.

Depre C, Young ME, Ying J, Ahuja HS, Han Q, Garza N, Davies PJ \& Taegtmeyer H 2000 Streptozotocin-induced changes in cardiac gene expression in the absence of severe contractile dysfunction. Journal of Molecular and Cellular Cardiology 32 985-996.

Dillmann WH 1989 Diabetes and thyroid hormone-induced changes in cardiac function and their molecular basis. Annual Review of Medicine 40 373-394.

Duan J, Zhang HY, Adkins SD, Ren BH, Norby FL, Zhang X, Benoit JN, Epstein PN \& Ren J 2003 Impaired cardiac function and IGF-I response in myocytes from calmodulin-diabetic mice: role of Akt and RhoA. American Journal of Physiology. Endocrinology and Metabolism 284 E366-E376.

Fein FS 1990 Diabetic cardiomyopathy. Diabetes Care 13 1169-1179.

Guo W, Kada K, Kamiya K \& Toyama J 1997 IGF-I regulates $\mathrm{K}(+)$-channel expression of cultured neonatal rat ventricular myocytes. American Journal of Physiology. Heart and Circulatory Physiology 272 H2599-H2606.

Hofmann PA, Menon V \& Gannaway KF 1995 Effects of diabetes on isometric tension as a function of $\left[\mathrm{Ca}^{2+}\right]$ and $\mathrm{pH}$ in rat skinned cardiac myocytes. American Journal of Physiology. Heart and Circulatory Physiology 269 H1656-H1663.

Isgaard J, Tivesten A, Friberg P \& Bengtsson BA 1999 The role of the GH/IGF-I axis for cardiac function and structure. Hormone and Metabolic Research 31 50-54. 
Kajstura J, Fiordaliso F, Andreoli AM, Li B, Chimenti S, Medow MS, Limana F, Nadal-Ginard B, Leri A \& Anversa P 2001 IGF-I overexpression inhibits the development of diabetic cardiomyopathy and angiotensin II-mediated oxidative stress. Diabetes $\mathbf{5 0}$ 1414-1424.

Karoor V \& Malbon CC 1996 Insulin-like growth factor receptor-1 stimulates phosphorylation of the beta2-adrenergic receptor in vivo on sites distinct from those phosphorylated in response to insulin. Journal of Biological Chemistry 271 29347-29352.

Lefroy DC, Crake T, Del Monte F, Vescovo G, Dalla Libera L, Harding S \& Poole-Wilson PA 1996 Angiotensin II and contraction of isolated myocytes from human, guinea pig, and infarcted rat hearts. American Journal of Physiology. Heart and Circulatory Physiology 270 H2060-H2069.

Leri A, Liu Y, Wang X, Kajstura J, Malhotra A, Meggs LG \& Anversa P 1999 Overexpression of insulin-like growth factor-1 attenuates the myocyte renin-angiotensin system in transgenic mice. Circulation Research 84 752-762.

Montgomery DE, Wolska BM, Pyle WG, Roman BB, Dowell JC, Buttrick PM, Koretsky AP, Del Nido P \& Solaro RJ 2002 $\alpha$-Adrenergic response and myofilament activity in mouse hearts lacking PKC phosphorylation sites on cardiac TnI. American Journal of Physiology. Heart and Circulatory Physiology 282 H2397-H2405.

Nishimaru K, Kobayashi M, Matsuda T, Tanaka Y, Tanaka H \& Shigenobu K $2001 \alpha$-Adrenoceptor stimulation-mediated negative inotropism and enhanced $\mathrm{Na}(+) / \mathrm{Ca}(2+)$ exchange in mouse ventricle. American Journal of Physiology. Heart and Circulatory Physiology $280 \mathrm{H} 132-\mathrm{H} 141$.

Norby FL, Wold LE, Duan J, Hintz KK \& Ren J 2002 IGF-I attenuates diabetes-induced cardiac contractile dysfunction in ventricular myocytes. American Journal of Physiology. Endocrinology and Metabolism 283 E658-E666.

Reiss K, Cheng W, Ferber A, Kajstura J, Li P, Olivetti G, Homcy CJ, Baserga R \& Anversa P 1996 Overexpression of insulin-like growth factor-1 in the heart is coupled with myocyte proliferation in transgenic mice. PNAS 93 8630-8635.

Ren J 2000 Altered cardiac contractile responsiveness to insulin-like growth factor I in ventricular myocytes from BB spontaneous diabetic rats. Cardiovascular Research 46 162-171.

Ren J \& Davidoff AJ 1997 Diabetes rapidly induces contractile dysfunctions in isolated ventricular myocytes. American Journal of Physiology. Heart and Circulatory Physiology 272 H148-H158.

Ren J \& Bode AM 2000 Altered cardiac excitation-contraction coupling in ventricular myocytes from spontaneously diabetic BB rats. American Journal of Physiology. Heart and Circulatory Physiology $279 \mathrm{H} 238-\mathrm{H} 244$.

Ren J \& Brown-Borg HM 2002 Impaired cardiac excitationcontraction coupling in ventricular myocytes from Ames dwarf mice with IGF-I deficiency. Growth Hormone and IGF Research 12 99-105.

Ren J, Samson WK \& Sowers JR 1999 Insulin-like growth factor I as a cardiac hormone: physiological and pathophysiological implications in heart disease. Journal of Molecular and Cellular Cardiology 31 2049-2061.

Ren J, Duan J, Hintz KK \& Ren BH 2003 High glucose induces cardiac insulin-like growth factor I resistance in ventricular myocytes: role of Akt and ERK activation. Cardiovascular Research 57 738-748.

Rodrigues B, Cam MC \& McNeill JH 1998 Metabolic disturbances in diabetic cardiomyopathy. Molecular and Cellular Biochemistry 180 53-57.

Roth DA, White CD, Hamilton CD, Hall JL \& Stanley WC 1995 Adrenergic desensitization in left ventricle from streptozotocin diabetic swine. Journal of Molecular and Cellular Cardiology 27 $2315-2325$.

Sakurai K, Norota I, Tanaka H, Kubota I, Tomoike H \& Endo M 2002 Negative inotropic effects of angiotensin II, endothelin-1 and phenylephrine in indo-1 loaded adult mouse ventricular myocytes. Life Sciences 70 1173-1184.

Samarel AM 2002 IGF-I overexpression rescues the failing heart. Circulation Research 90 631-633.

Sekine T, Kusano H, Nishimaru K, Tanaka Y, Tanaka H \& Shigenobu K 1999 Developmental conversion of inotropism by endothelin I and angiotensin II from positive to negative in mice. European Journal of Pharmacology 374 411-415.

Solem ML \& Thomas AP 1998 Modulation of cardiac $\mathrm{Ca}^{2+}$ channels by IGF-I. Biochemical and Biophysical Research Communications 252 151-155.

Wang L, Ma W, Markovich R, Chen J-W \& Wang PH 1998 Regulation of cardiomyocyte apoptotic signaling by insulin-like growth factor. Circulation Research 83 516-522.

Welch S, Plank D, Witt S, Glascock B, Schaefer E, Chimenti S, Andreoli AM, Limana F, Leri A, Kajstura J, Anversa P \& Sussman MA 2002 Cardiac-specific IGF-I expression attenuates dilated cardiomyopathy in tropomodulin-overexpressing transgenic mice. Circulation Research 90 641-648.

Ye G, Metreveli NS, Ren J \& Epstein PN 2003 Metallothionein prevents diabetes-induced deficits in cardiomyocytes by inhibiting reactive oxygen species production. Diabetes $\mathbf{5 2} 777-783$.

Received 21 August 2003

Accepted 18 September 2003

Made available online as an

Accepted Preprint 24 September 2003 